CRÍTICA, Revista Hispanoamericana de Filosofia

Vol. XXIX, No. 86 (agosto 1997): 53-81

\title{
DE LA RACIONALIDAD A LA RAZONABILIDAD: ¿ES POSIBLE UNA FUNDAMENTACIÓN EPISTEMOLÓGICA DE UNA MORAL "POLÍTICA"?
}

\author{
EdUARDo Rivera LóPEZ \\ Universidad de Buenos Aires \\ Universidad Torcuato Di Tella
}

\begin{abstract}
¿Cree que es importante el movimiento liberal de la Iglesia Católica?

Creo que el movimiento actual de la Iglesia Católica corresponde a una debilidad. Cuando la Iglesia era fuerte, no era tolerante; cuando la Iglesia era fuerte quemaba y perseguía. Pero creo que en una buena parte la tolerancia de la iglesia corresponde a una debilidad, y no porque sea mas broadmind$e d$, porque no puede serlo. Las iglesias - cualquiera que sea, católica, protestante - no han sido tolerantes. Si yo creo estar en posesión de la verdad, no tengo por que ser tolerante con aquellos que estan arriesgando su salvación creyendo en errores; al contrario, casi tengo la obligación de perseguirlos. No puedo decir: "No es importante que usted sea protestante porque al final todos somos hermanos de Cristo." No. Decir eso ya es muestra de escepticismo.
\end{abstract}

Jorge Luis Borges ${ }^{1}$

El pensamiento de John Rawls, en sus diversas etapas, ha tenido un rasgo poco frecuente, dentro de la filosofía política de corte analítico: independientemente del enorme valor específico de las diferentes argumentaciones que ha desarrollado, ha tenido la virtud de instalar en el debate fi-

1 De un reportaje aparecido en Jaime Alazraky (comp.), Jorge Luis Borges, Taurus, Madrid, 1984, p. 354. 
losófico un conjunto de "ideas-fuerza", que luego nadie ha podido pasar por alto. Ideas como la de la posición original, velo de la ignorancia, principio de diferencia, han llenado (y siguen llenando) páginas de discusión, sobre la base de la primera formulación de su teoría. Hoy, nos vemos obligados a recurrir a conceptos y distinciones tales como doctrina moral comprehensiva y política, consenso sobrepuesto, dificultades del juzgar, razonabilidad, etc. Esto es, insisto, independiente de las soluciones concretas ofrecidas por Rawls; se trata de ideas que nos obligan a plantear de otro modo y con otros conceptos los problemas éticopolíticos de siempre.

Mi propósito en este trabajo es plantear el problema ("de siempre") de la fundamentación moral de ciertos deberes básicos que todo individuo (y el Estado) asume en una sociedad, en términos generales, liberal. Centralmente, me interesa argumentar en favor del deber de tolerancia o respeto por concepciones del bien diversas. Lo haré utilizando muchas de las distinciones y conceptos propuestos por Rawls en Political Liberalism, aunque el argumento, en sí mismo, difiere en puntos esenciales de la propuesta concreta de Rawls en ese libro.

La primera idea a la que quisiera aludir, y que resulta recurrente dentro de la obra de Rawls, es la distinción entre racionalidad y razonabilidad. La racionalidad consiste en la capacidad de proponerse fines, estilos y planes de vida, y de disponer los medios para satisfacerlos de un modo eficiente. La razonabilidad se identifica, en Rawls, con el sentido moral, es decir, con la capacidad para concebir a los otros individuos como libres e iguales y entablar con ellos una relación de cooperación sobre bases equitativas. ${ }^{2}$ Rawls enfatiza la idea de que ambas capacidades deben considerarse

${ }^{2}$ Véase Rawls 1993, pp. 49-50. Para una reconstrucción excelente del concepto de "persona razonable" en Rawls, véase Wenar 1995, p. 37. Wenar no incluye explícitamente el hecho de que personas 
capacidades morales, no solamente la razonabilidad (que es obviamente moral). La racionalidad, para Rawls, no se circunscribe a una racionalidad puramente instrumental, sino que consiste también en dar valor a los fines que se persiguen, considerándolos como algo “bueno". Más aún, si bien Rawls no lo afirma explícitamente, puede pensarse que la racionalidad es la capacidad que permite a los individuos concebir o participar de, o adherir a, una "concepción comprehensiva" moral, filosófica o religiosa.

Sobre la base de esta distinción, la pregunta que surge, a mi juicio, naturalmente, es: ¿̨cómo es posible pasar de la mera racionalidad a la razonabilidad? $\mathrm{O}$, dicho de otro modo, ¿cómo es posible que individuos que poseen concepciones comprehensivas diferentes, e incluso opuestas, estén dispuestos a aceptar términos de cooperación equitativos y a ser tolerantes (i.e. no pretendan reprimir aquellas concepciones comprehensivas diferentes a la propia)? Es decir, ¿cómo pasar de una mera concepción comprehensiva a una concepción comprehensiva razonable? ${ }^{4}$

Sin embargo, Rawls no plantea la pregunta de este modo. Su pregunta es: “¿Cómo es posible que exista a través del tiempo una sociedad justa y estable de ciudadanos libres

razonables se consideran mutuamente libres e iguales, pero es claro que lo presupone. La siguiente cita de Rawls lo muestra claramente: "Las personas son razonables en un aspecto básico cuando, entre iguales, estan dispuestos a proponer principios y estándares como cooperación equitativa [...]." (Rawls 1993, p. 49.) También: “decimos que las personas razonables no están motivadas por el bien general como tal, sino que desean por sí mismo un mundo social en el que ellos, como libres e iguales, pueden cooperar con otros en términos que todos pueden aceptar." (Rawls 1993, p. 50.)

3 Véase Rawls 1993, pp. 50-51.

4 Nótese que esta pregunta no puede identificarse con el propósito de Gauthier, que Rawls rechaza explícitamente (Rawls 1993, p. 51), dado que lo racional esta definido de un modo mucho más amplio y rico que en la teoría de Morals by Agreement. 
e iguales, que, sin embargo, permanecen profundamente divididos por doctrinas religiosas, filosóficas y morales $\mathrm{ra}$ zonables?". 5 Incluir la propiedad de la razonabilidad en la pregunta lleva, a mi juicio, a una peligrosa circularidad. Si se presupone que los individuos que permanecen profundamente divididos por sus doctrinas comprehensivas son razonables, entonces no resulta sorprendente que puedan coexistir a través del tiempo dentro de una sociedad justa y estable. Lo que otorga los valores de tolerancia, estabilidad y cooperación equitativa es precisamente la razonabilidad, dado que una concepción comprehensiva razonable, según Rawls, es, precisamente aquella capaz de concordar parcialmente con otras concepciones razonables, conformando un "consenso sobrepuesto". La teoría que emane de ese consenso ya no será una doctrina comprehensiva, porque no abarcará todas las facetas de la moralidad, sino política, en el sentido de que regulará la convivencia entre las diferentes doctrinas comprehensivas en el ámbito público de discusión. Estas doctrinas comprehensivas pasarán a ser, por tanto, doctrinas comprehensivas razonables.

Por supuesto, una evaluación definitiva de la estrategia de Rawls merecería un análisis mas detallado. Como adelanté, no es mi propósito llevar a cabo esta tarea. Sin excluir la posibilidad de que la estrategia rawlsiana pueda ser fructífera, quisiera utilizar algunos de sus elementos conceptuales y distinciones para explorar una posible respuesta a la pregunta básica, tal como la he formulado inicialmente (es decir, sin incluir el elemento de la razonabilidad como presupuesto). La misma tendrá la forma de una argumentación en cuatro pasos, con el objetivo de dar razones a favor de una teoría "política" tolerante, partiendo de premisas que, en lo posible, no incluyan, ni explícita ni implícitamente,

5 Rawls 1993, p. 47, las cursivas son mías. 
los valores de la razonabilidad, la tolerancia, la libertad e igualdad entre los individuos o el sentido de la justicia.

\section{I}

En primer lugar, quisiera retomar la distinción trazada anteriormente. Por un lado, podemos concebir el conjunto de posibles doctrinas comprehensivas morales, filosóficas o religiosas. Estas "doctrinas" pueden incluir teorías articuladas acerca de lo que es bueno, correcto, debido, valioso como fin, etc., o, simplemente, ideas intuitivas y no explicitadas al respecto. En este sentido, podemos decir que todo individuo mínimamente racional posee o adhiere a cierta doctrina comprehensiva. Todo individuo adhiere con mayor o menor convencimiento y grado de racionalización, por ejemplo, a ciertas ideas acerca de la religión (p.ej., es religioso o no lo es), ciertas pautas morales (p.ej., cree que matar es malo o que no lo es, que no se debe mentir o engañar o que se puede hacerlo, etc.), ciertos fines que cree valioso alcanzar (fines de realización personal o comunitaria, por ejemplo), etc. Por otro lado, podemos imaginar el conjunto de las posibles teorías "políticas". Éstas se refieren solamente a cómo diferentes doctrinas comprehensivas pueden o deben tratarse entre sí. O, dicho con mas precisión: cómo los individuos que encarnan doctrinas comprehensivas diferentes e incompatibles deben tratarse entre sí, de qué modo deben interaccionar. Como dije anteriormente, el problema que deseo tratar es el de dar razones en favor de una determinada teoría "política" en este sentido.

Una primera característica fácilmente comprobable del primer nivel (el de las doctrinas comprehensivas) es la enorme dificultad para dar razones concluyentes en favor de alguna doctrina moral comprehensiva. El origen de esta dificultad se encuentra en las, así llamadas por Rawls, "dificultades del juzgar" (burdens of judgment). Estas di- 
ficultades del juzgar pueden interpretarse, simplemente, como la transferencia al plano de la moral de una idea ya largamente aceptada por la epistemología en el plano de la ciencia: que es enormemente difícil verificar, o incluso solamente corroborar enunciados con contenido (es decir, no analíticos), especialmente si se trata de enunciados universales. ${ }^{6}$ No explicitaré detalladamente la lista que presenta Rawls, pero, para recordar los diferentes tipos de dificultades, menciono los más importantes: la dificultad de evaluar, y sopesar la relevancia de evidencias empíricas, la vaguedad de los conceptos morales y políticos (necesidad de interpretación), la influencia de nuestra experiencia vital total sobre nuestros juicios morales y políticos concretos, la existencia de conflictos o dilemas morales, etc. ${ }^{7}$ Estas dificultades bastante obvias no deben llevar necesariamente a sostener que es inútil discutir ideas o propuestas de doctrinas morales comprehensivas, del mismo modo que tiene sentido discutir acerca de diferentes teorías científicas, a pesar de las enormes dificultades con que se enfrenta la tarea de confirmar teorías o hipótesis. Pero esta discusión, es necesario admitir, está restringida por fuertes limitaciones en la posibilidad de llegar a conclusiones firmes o resultados concluyentes.

Este argumento, basado en las dificultades del juzgar, explica el hecho del pluralismo de doctrinas comprehensivas. No se trata, todavía, de un pluralismo razonable, sino de un mero pluralismo, o, para utilizar un término no cargado de connotaciones morales, de una pluralidad de doctrinas comprehensivas. ${ }^{8}$ Este "hecho" no debe interpretarse simplemente como un dato histórico de alguna sociedad (como

${ }^{6}$ Al respecto, sigo la idea ya clásica de Popper 1961, §3.

7 Véase Rawls 1993, pp. 56-58.

${ }^{8}$ Aquí, nuevamente, me aparto de Rawls. Él sostiene que las dificultades del juzgar explican el pluralismo razonable: "La idea del desacuerdo razonable incluye una explicación de las fuentes, o causas 
la nuestra) sino que posee una generalidad más interesante. Así como el pluralismo de teorías científicas no es solamente un hecho en la historia de la ciencia, sino un hecho que se infiere de la imposibilidad de verificar hipótesis científicas, la pluralidad de concepciones morales, filosóficas, religiosas, es un hecho que se infiere de las dificultades del juzgar, es decir, en última instancia, de las limitaciones y condicionamientos del conocimiento humano. En otras palabras: siempre existirá, al menos potencialmente, una pluralidad de doctrinas comprehensivas. Incluso si se da la circunstancia empírica de que durante cierto periodo de la historia una concepción resulte dominante (como el caso de la concepción cristiana durante la Edad Media), es posible afirmar que tal circunstancia tarde o temprano entrará en crisis y otras concepciones competirán con la dominante. ${ }^{9}$ La enorme dificultad de los problemas morales, la enorme cantidad de preguntas abiertas que toda concepción moral o religiosa comprehensiva deja abierta (al igual que toda teoría científica) hace que continuamente surjan nuevas posibles respuestas, nuevos intentos, nuevas conjeturas.

del desacuerdo entre personas razonables. A estas fuentes las llamo las dificultades del juzgar." (Rawls 1993, p. 55.) Yo creo que las dificultades del juzgar explican el hecho del pluralismo a secas, es decir, el hecho de que haya diferentes doctrinas comprehensivas racionales, y no razonables. Creo que las dificultades del juzgar no deberían incluirse dentro del concepto de persona razonable, porque no contienen, en sí mismos, ninguna connotación moral. Simplemente afirman que es difícil hallar respuestas concluyentes en temas morales, religiosos, etc., y que esto explica que haya diversas doctrinas. Dicho de otro modo, si fuera evidente para cualquiera cuál es la posición moral o religiosa correcta, no habría tal pluralismo: todo el mundo adheriría a ella.

9 En este punto sigo a Rawls, aunque de un modo algo más débil. Él sostiene que la inquisición no era un elemento accidental sino imprescindible para mantener la uniformidad doctrinaria en la Edad Media. No sé si esto es tan exacto históricamente. Yo solamente sostengo que no es accidental que en algún momento haya tenido que aparecer la inquisición, si se pretendía continuar manteniendo esa uniformidad. 
Sobre la base de estos argumentos, es plausible pensar que un individuo racional que comparte, adhiere o practica una determinada doctrina comprehensiva será capaz de aceptar que la razón de la existencia de la pluralidad es la existencia de las dificultades del juzgar y tomará en cuenta seriamente estas dificultades para evaluar también su propia doctrina.

Una persona racional tomará en cuenta las dificultades del juzgar. Esto lo llevará, en primer lugar, a utilizarlas para explicar el hecho de la pluralidad de doctrinas comprehensivas.

Ahora bien, el hecho de que exista esta pluralidad, esta diversidad, este desacuerdo, no quiere decir que no podamos defender una doctrina moral comprehensiva determinada. Podemos, por ejemplo, ser católicos, o liberales, o marxistas, o utilitaristas, o hedonistas, etc. Ahora bien, las dificultades del juzgar adquieren en este contexto otro papel, dentro de cada doctrina comprehensiva. En efecto, si, además de ser católicos, o liberales, o marxistas, admitimos la existencia de las dificultades del juzgar y le otorgamos el peso adecuado, entonces creo que no podemos ser cualquier tipo de católico, liberal o marxista. Debemos admitir la posibilidad de estar equivocados. Aunque creamos fuertemente en nuestra doctrina, debemos dejar un resquicio de duda. Rawls defiende, al respecto, una posición diferente. ${ }^{10}$ Él considera que cada uno puede creer firmemente en la verdad de su concepción comprehensiva, pero aceptar que las otras también son razonables.

Creo que éste es un punto central de la argumentación que deseo proponer y, por ello, un punto bastante delicado. Mi argumento al respecto es el siguiente. Toda doctrina

10 Véase Rawls 1993, p. 61. 
comprehensiva puede descomponerse conceptualmente en (al menos) dos niveles: por un lado, un conjunto de afirmaciones, generalmente normas, pautas de conductas, pero también proposiciones (por ejemplo, del tipo "Dios existe", o "Dios no existe", etc.); por otro, un conjunto de proposiciones acerca del grado de credibilidad del primer conjunto. Éstas son, por así decir, creencias epistemológicas, que nos dicen cuán seguros podemos estar acerca de la verdad o justificabilidad de las afirmaciones del primer nivel. No es importante aquí si de hecho las doctrinas comprehensivas corrientes poseen este segundo tipo de afirmaciones. Podemos suponer que, o bien las contienen (aunque sea mezcladas con las otras), o bien el individuo que cree o participa de esa doctrina tendrá que tener alguna opinión al respecto (por ejemplo, creerá firmemente, o tendrá dudas, etc.). Muchas veces las afirmaciones epistemológicas no estan explícitas sino sólo sugeridas y, por tanto, diferentes adherentes a una misma doctrina difieren respecto de su grado de certeza o interpretan de modo diferente esas sugerencias (por ejemplo, diferentes católicos tienen creencias epistemológicas diferentes acerca de los dogmas de la religión católica). Para expresar de un modo claro lo que deseo defender, diré que un individuo racional que, por tanto, toma en cuenta seriamente las dificultades del juzgar, tendrá una posición de no total certeza en el plano epistemológico. En otras palabras, será un falibilista en materia moral. ¿Por qué? Porque no existe ninguna razón para pensar que estas dificultades sólo alcanzan a las doctrinas de los otros pero no a la propia. La propia doctrina también se ve afectada por las dificultades del juzgar. Ella también está limitada por estas dificultades. Sobre ella también pesa la enorme dificultad de probar los enunciados teóricos sustantivos.

Por supuesto, en el segundo nivel de creencias (las creencias "epistemológicas") podrá haber grados. Por ello, he 
dicho que algunos podrán adherir a una doctrina más firmemente que otros (que aquellos que tienen dudas profundas). El falibilismo moral lo único que afirma es que existe un límite máximo de credibilidad en la propia doctrina comprehensiva, que es más bajo que el de la verificación o prueba concluyente. Esto significa que, aun aquellos que creen firmemente, tendrán que admitir que pueden estar equivocados.

Esta posición falibilista no implica, debe quedar claro (aquí sí concuerdo con Rawls), ser un escéptico moral. ${ }^{11}$ No implica, por ejemplo, creer que no puede existir una fundamentación objetiva de las normas morales, o las creencias religiosas. Creo, más bien, que el escepticismo moral debe considerarse, en realidad, como una de las posibles doctrinas morales comprehensivas. ${ }^{12}$ En este sentido, el escéptico moral también debería tomar su posición escéptica como una hipótesis o conjetura teórica. La afirmación de que no existe una justificación objetiva de las normas y éstas, por tanto, sólo poseen una validez relativa, también se encuentra limitada y condicionada por las dificultades del juzgar. ${ }^{13}$

11 Al respecto, véase Rawls 1993, pp. 62-63.

12 Esta idea no debe confudirse con la defendida recientemente por Dworkin en 1996. Dworkin sostiene allí que toda teoría metaética (y el escepticismo pretende ser, centralmente, una tesis metaética) es, cuando se la interpreta correctamente, una tesis moral más, una tesis sustantiva. Mi idea es algo diferente: puede ser que el escepticismo moral sea una tesis metaética, pero todo escéptico moral verosímil deberá ser, en el plano epistemológico, un falibilista (sería muy poco creíble un escéptico moral que fuera dogmático en el nivel epistemológico). Por lo tanto, deberá tomar sus tesis epistemológica escéptica como una hipótesis que está sometida también a las dificultades del juzgar.

13 Ciertamente, los juicios de inexistencia, por razones lógicas, son mucho más difíciles de verificar. Por ejemplo, la proposición “no existen centauros" es (en un universo infinito) inverificable, mientras que "existen centauros" se puede probar señalando la existencia de uno solo. Por ello, se supone normalmente que la "carga de la prueba" para 
Lo único que las dificultades del juzgar llevan a creer es que no existe ninguna doctrina moral comprehensiva que pueda pretender el título de teoría verificada, de teoría probada de manera concluyente. Nuevamente, el paralelo con la ciencia es, creo, iluminador. El hecho de reconocer las dificultades de la confirmación científica no lleva, necesariamente, a ser escéptico desde el punto de vista del conocimiento teórico. Sólo lleva a considerar a las teorías como conjuntos de hipótesis no verificadas, o, en el mejor de los casos, corroboradas.

De este modo, completamos el segundo paso de la argumentación: tomar en serio las dificultades del juzgar lleva a adoptar una posición epistemológica falibilista respecto de la propia doctrina moral comprehensiva. Para simplificar, llamaré a esta posición "falibilismo ético" (o, a veces, simplemente, "falibilismo"). ${ }^{14}$

determinar si algo existe o no, la lleva el que sostiene la existencia (él tiene la tarea más fácil). En este sentido, cuando el ateo sostiene que no hay pruebas de la existencia de Dios, no es admisible la respuesta (bastante habitual): "usted tampoco probó que no existe". Sin embargo, en un contexto de discusión en el que se dan argumentos tanto a favor como en contra de la existencia de algo, el que sostiene la inexistencia también debe ofrecer argumentos positivos que lo llevan a creer que no hay razones para admitir la existencia de la cosa en cuestión (y, de hecho, existen argumentos a favor de la inexistencia de Dios). Y estos argumentos están sometidos, como todos, a las dificultades del juzgar.

14 En este punto, mi posición se parece a la de Brian Barry 1995. Él distingue dos niveles que toda concepción comprehensiva debería poseer: un primer nivel de creencias que pueden ser dogmáticas (por ejemplo, creer firmemente en Dios), y un segundo nivel "epistemológico" que debe ser "escéptico" (dado que acepta las dificultades del juzgar) para que pueda ser razonable (véase Barry 1995, p. 901). Yo no estoy seguro de que el escepticismo en el nivel epistemológico (que yo denominaría "falibilismo") no tenga consecuencias en el nivel del contenido: ¿puedo creer firmemente, como algo totalmente irrefutable, que fuera de la Iglesia no es posible la salvación, y, simultáneamente, creer que el status epistemológico de esa creencia es el de una creencia dudosa? 
Ahora bien, sobre la base de lo dicho, es claro que podemos distinguir dos posiciones epistemológicas frente a una doctrina comprehensiva cualquiera: la que incluye la posibilidad de que la doctrina esté errada (es decir, la que la considera un conjunto de hipótesis o conjeturas acerca de la moral o la religión o la vida buena), y la que no. Es decir, la posición que toma seriamente en cuenta las dificultades del juzgar y la que no lo hace. Esta última, es decir, la posición que (por contraste con el falibilismo ético) podemos denominar "dogmatismo ético", que no admite la menor duda acerca de la verdad de la doctrina, puede, a su vez, dar lugar a dos tipos de actitudes o razones para actuar. Por un lado, puede pensarse en una posición dogmática acerca de una doctrina que, si bien considera terminantemente falsas todas las doctrinas comprehensivas incompatibles con la propia, produzca una actitud tolerante para con ellas. El contenido de la doctrina debe ser tal que no implique la obligación de forzar a los demás a su obediencia. Por otro, puede pensarse en una posición dogmática para la cual el hecho de que otras doctrinas estén equivocadas haga surgir una razón suficiente para querer suprimirlas. No existe ningún criterio externo a las doctrinas acerca de las cuales se toma una posición dogmática para decidir cuándo éstas producen una actitud tolerante o no. Esto depende de modo contingente del contenido de cada una de ellas. ${ }^{15}$ Por ello, podemos llamarlas, respectivamente, "actitud internamente tolerante" y "actitud intolerante". Por otro lado, tenemos la posición "falibilista ética", es decir, la que presenta la doctrina sólo como una

15 Utilizando la distinción en dos niveles mencionada con anterioridad, se podría decir que depende contingentemente de las afirmaciones del primer nivel, es decir, del nivel del contenido. Depende también, naturalmente, de la interpretación que cada individuo haga de ese contenido. 
hipótesis (conjunto de hipótesis) acerca de la fundamentación y contenido de la moralidad, de lo que es valioso, correcto, etc., pero que no pretende excluir la posibilidad de estar parcial o totalmente equivocada.

El tercer paso de mi argumentación consiste, entonces, en sostener que la posición falibilista ofrece razones muy poderosas para asumir una actitud tolerante respecto de otras doctrinas, así como para establecer límites a esa tolerancia. ${ }^{16}$ Estas razones no son internas o dependientes del contenido de la doctrina, como sucede en el caso de la posición dogmática, sino que se siguen de su carácter hipotético. Este tercer paso es, sin duda, el paso clave y más riesgoso, dado que debemos extraer consecuencias morales a partir de algo que, en sí mismo, no es moral, a saber, un falibilismo acerca de la moral. Mi tesis consiste, entonces, en sostener que, si aceptamos el falibilismo ético, es decir, si aceptamos que nuestra concepción moral comprehensiva puede estar equivocada, entonces tenemos buenas razones para ser repetuosos con otras concepciones morales comprehensivas. Una premisa importante del argumento es que, si bien no nos creemos con la verdad en la ma-

16 Una aclaración sobre el término tolerancia. Creo que posee una connotación doble. Por un lado, tolerar quiere decir respetar, no querer suprimir una doctrina o punto de vista diferente al nuestro. Por otro, la actitud de tolerancia implica una restricción hacia uno mismo: se tolera a quienes no piensan como uno y a los que, en principio, tenderíamos a no permitir que lo hagan. A pesar de que no están de acuerdo con nosotros, aceptamos que existan. Ambos aspectos (el de mero respeto y el de autorrestricción) creo que se corresponden muy bien con la idea que deseo expresar en mi argumento. Cada individuo abraza una doctrina moral comprehensiva diversa, y debemos argumentar por qué, a pesar de ello, debemos poseer una actitud de respeto. Ciertamente, una vez que este paso argumentativo se ha llevado a cabo, y hemos aceptado la actitud tolerante, ya no tiene sentido continuar hablando de tolerancia, sino, directamente, de derechos (derecho de libre conciencia, de libre expresión, de libre pensamiento, derechos políticos, etc.). 
no, estamos preocupados por encontrar la verdad. Asumo, en este sentido, que, dado el papel central que las ideas morales y religiosas desempeñan en nuestras vidas (cualquiera que sea nuestra posición frente a ellas), cualquier individuo racional está interesado en hallar o acercarse a la doctrina moral o religiosa correcta. Las decisiones acerca de nuestra religión (si seremos religiosos o no, y de qué manera), acerca de nuestros fines básicos, acerca del modo en que perseguiremos esos fines, etc. son fundamentales para poder afirmar que llevamos una vida "buena". Por ello, la percepción de que tomamos decisiones correctas en esos ámbitos es indispensable para otorgar sentido a nuestra vida. Por otro lado, estamos forzados a tomar este tipo de decisiones, que, por lo demás, no podemos declarar tan fácilmente como triviales o intrascendentes. Por ello, creo que no es arriesgado afirmar que la búsqueda de una doctrina moral o religiosa verdadera, es una búsqueda racional. Si este interés es racional, y, además, aceptamos el falibilismo moral, entonces creo que tendremos muy buenas razones para ser tolerantes. Para ilustrar este punto, recurro nuevamente al paralelo con la ciencia. Si aceptamos que las teorías científicas son conjuntos de hipótesis o conjeturas, pero estamos interesados en encontrar la teoría verdadera, tenemos buenas razones para escuchar atentamente teorías adversarias a la nuestra, y, por lo tanto, que se consagre un derecho a exteriorizar y escuchar opiniones científicas.

De este modo, podemos llamar a la actitud que se sigue, a mi juicio, del falibilismo ético e infiere de ello la tolerancia, actitud "externamente tolerante". ${ }^{17}$ Es externa

17 De este punto de vista se desprende una crítica a Rawls, que es exactamente opuesta a la que le realiza Leif Wenar en 1995. Él afirma que las dificultades del juzgar son innecesarias, porque se puede ser tolerante sin aceptarlas, y excluyentes, porque existen doctrinas (como la de la Iglesia Católica) que son tolerantes y explícitamente las rechazan (típicamente, no explican la pluralidad de doctrinas religiosas 
porque la actitud tolerante (a diferencia del dogmático, que posee una actitud internamente tolerante) no depende del contenido de la doctrina a la que se adhiere.

Es necesario ahora aclarar con mayor precisión cuál es el objeto de la actitud (externamente) tolerante. En el argumento presentado aparecen indistintamente como destinatarios de la actitud tolerante las concepciones mismas (en el sentido de tolerar que las personas posean determinadas creencias) y las prácticas que se siguen de esas creencias. Ambas cosas son, en principio, diferentes. Se podría sostener que uno puede ser tolerante respecto de las teorías y no serlo de las prácticas o viceversa. Por ejemplo, uno podría permitir que alguien sostenga o crea una determinada doctrina, pero prohibir algunas o todas las prácticas que se siguen de ella. $\mathrm{O}$, en el segundo caso, uno podría afirmar que una concepción X es, con toda certeza, falsa, pero, sin embargo, permitir que aquellos que participan de ella la practiquen en paz. Ambas alternativas se refieren a diferentes modos de ser tolerante o intolerante con las personas. En el primer caso, cuando se toleran las creencias y no las prácticas y, en el segundo, cuando se toleran las prácticas y no las creencias. Lo primero no es muy frecuente, pero no es imposible: puede tolerarse que las personas sean, por ejemplo, marxistas (en el sentido de que crean en el marxismo, lean sus textos, etc.) pero no tolerar ninguna práctica que se origine en esa doctrina (digamos, fundar un

por las dificultades del juzgar sino por las tentaciones del diablo, la libre determinación humana o cosas similares) (véase Wenar 1995, pp. 4148.) Mi opinión, en cambio, es que un individuo será externamente tolerante si acepta las dificultades del juzgar y, además, extrae de allí un falibilismo ético (cosa que Rawls no hace: él cree que se pueden aceptar las dificultades del juzgar y estar totalmente seguro de la verdad de la propia doctrina). Si no acepta las dificultades del juzgar (o no extrae de allí el falibilismo ético), entonces podrá ser internamente tolerante, como lo es, actualmente (aunque no monolíticamente) la Iglesia Católica. 
partido comunista, organizar el proletariado, etc.). Lo segundo es más frecuente: muchos de nosotros estamos completamente seguros de que alguna religión afro-brasilera es completamente absurda e indefendible, pero, sin embargo, creemos que hay que tolerar sus prácticas (mientras no sobrepasen ciertos límites).

Por tanto, la actitud tolerante que, según mi argumento, una persona racional adoptaría, en virtud de su falibilismo ético, tiene por destinatarios a las personas en dos niveles, el nivel de sus prácticas o conductas y el nivel de sus creencias.

La primera objeción que se puede realizar a mi argumento es que éste únicamente prueba que un individuo que ha adoptado el falibilismo ético tendría razones para ser tolerante solamente en el primer nivel, es decir, respecto de las creencias, pero no tendría razones suficientes para serlo respecto de las conductas o prácticas. Si mi interés es el de encontrar la doctrina moral comprehensiva correcta tendré razones para permitir el desarrollo teórico de doctrinas diferentes a la mía, pero no necesariamente para permitir que otros practiquen esas doctrinas o realicen conductas acordes con ellas.

Sin embargo, creo que es posible mostrar que esta actitud sería altamente problemática. Nuevamente, puede ayudarnos la analogía con la ciencia. Tolerar creencias, sin tolerar conductas que se siguen de ellas, sería, análogamente, tanto como permitir los desarrollos teóricos de una teoría científica y prohibir que se realizaran los experimentos que la testean. De algún modo, el tipo de vida que lleva una persona de acuerdo con su concepción comprehensiva es una suerte de experimento (en el sentido de Mill). ${ }^{18} \mathrm{Ob}$ servamos cómo vive, cuán feliz o infeliz es, cuán exitosa o

18 En el caso de Mill, los diferentes modos de vida son experimentos que contribuyen al progreso moral. Véase Mill 1970, cap. 2. 
fracasada es, etc., la gente que piensa diferente de nosotros, $y$, en cierta medida, juzgamos su manera de pensar en virtud del resultado que esa manera de pensar tiene sobre sus vidas. Las doctrinas comprehensivas tienen una dimensión práctica ineliminable, por lo que sería imposible formarnos un juicio crítico completo acerca de una concepción moral o religiosa si no permitiéramos también que las personas que adhieren a ella la practiquen y actúen de acuerdo con ella.

Una segunda objeción se refiere al hecho frecuente de que (como ya he señalado) somos tolerantes (o, creemos que debemos serlo) respecto de prácticas, a pesar de rechazar absolutamente las creencias que las sustentan. En este sentido, se podría argüir que mi argumento, o bien debería tomar en serio todas las concepciones morales y religiosas, aun las más absurdas, o bien, no podría ser tolerante con las conductas que se derivan de las doctrinas rechazadas. Dicho de otro modo: si nuestro falibilismo ético tiene un límite y, digamos, aceptamos como claramente absurda e increíble la doctrina (religiosa o moral) X, entonces ya no tendríamos interés en observar las prácticas acordes con ella, dado que no nos proporcionaría nada interesante en nuestra búsqueda de la doctrina correcta. Por lo cual, no tendríamos razones para tolerarla, ni en el plano de la creencia, ni en el de las conductas, aun cuando $\mathrm{X}$ fuera inofensiva, por lo cual nuestro sentido común tendería a tolerarla. $\mathrm{O}$, en todo caso, no tendríamos una razón externa, y, por lo tanto independiente, para tolerarla, sino sólo dependiente del contenido de nuestra propia doctrina moral comprehensiva. Esta objeción cobra todavía más fuerza, si observamos qué ocurre con el falibilismo en el caso de la ciencia. Como es sabido, la posición falibilista poppereana sostiene la llamada "asimetría entre la verificación y la refutación". Esto significa que, mientras que no es posible verificar las hipótesis, sí es posible refutarlas. Y 
Popper presenta el camino de la ciencia, justamente, como determinado por refutaciones. Su metodología "normativa" propugna no intentar salvar teorías de la refutación, sino intentar refutarlas para mostrar su "temple". 19

Hay al menos tres argumentos que pueden debilitar en gran medida esta crítica. En primer lugar, debe tenerse en cuenta que, incluso en el plano de la ciencia, y aun cuando existe la posibilidad de la contrastación empírica de las afirmaciones singulares (cosa que en el caso de la moral no se da), la posibilidad de la refutación definitiva de una teoría es más una posibilidad lógica que real. ${ }^{20}$ En el campo de las teorías morales y religiosas, esta posibilidad es todavía más remota. En segundo lugar, las doctrinas comprehensivas no son bloques compactos que se toman en su totalidad o se rechazan en su totalidad. Una doctrina religiosa, por ejemplo, puede parecer totalmente absurda, porque sus ritos o algunas de sus creencias lo son. Pero la actitud religiosa en general (que tal religión necesariamente contendrá) no es absurda ni rechazable concluyentemente. En tercer lugar, existe un argumento, a mi juicio, poderoso, similar a uno de los argumentos a favor de ciertas formas de utilitarismo (o consecuencialismo) de regla o indirecto. Si la posición no dogmática respecto de la moral nos lleva a una actitud externamente tolerante, es muy difícil abandonar esta actitud en algunas ocasiones, sin que se frustre la actitud en su totalidad. Nuestra actitud tolerante nos

19 Para todo esto, véase Popper 1961, § 85.

20 La razón es, como es sabido, que la refutación de una teoría compleja (que consta de muchas hipótesis) significa que la conjunción de todas sus hipótesis es falsa (cfr. Popper 1961, §18). Ahora bien, una conjunción es falsa cuando sólo uno de sus miembros lo es. Esto quiere decir que un testeo empírico puede refutar una teoría, pero una revisión de sus hipótesis (por ejemplo, de sus hipótesis auxiliares) puede hacerla "revivir". Hay ejemplos clásicos en la historia de la ciencia de teorías refutadas que, con variaciones más o menos grandes, fueron retomadas. 
llevará a apoyar y promover reglas de conducta (e incluso reglas jurídicas) de protección de las diferentes doctrinas comprehensivas. Estas reglas se verían frustradas si, luego, intentáramos hacer excepciones sobre la base del contenido de alguna doctrina particular.

\section{IV}

Resumiendo lo argumentado hasta aquí: un individuo racional, que defiende una doctrina comprehensiva particular deberá tomar seriamente las dificultades del juzgar y aplicarlas a su propia doctrina. Esto lo llevará a sostener un falibilismo acerca de las doctrinas (en particular acerca de la suya). A su vez, esto le dará razones para adoptar una actitud externamente tolerante (es decir, una actitud tolerante no basada en el contenido concreto de su doctrina) hacia las doctrinas de los otros.

Ahora bien, un individuo con una actitud externamente tolerante deberá establecer los límites y el contenido normativo concreto de la tolerancia. Su principio de tolerancia no es absoluto sino prima facie. Existen casos en que deberá establecer restricciones. Un límite que parece claro es la exigencia de reciprocidad: sólo tiene sentido ser tolerantes con aquellos individuos que sean tolerantes con uno. Ser tolerantes, bajo la interpretación que he propuesto, quiere decir, estar dispuesto a escuchar otras opiniones en la búsqueda de la doctrina moral correcta y no interferir en las conductas y prácticas que surgen de una doctrina moral determinada. Pero escuchar a los demás o tolerar sus prácticas no implica creer que nuestra doctrina moral no tiene ningún valor; por el contrario, la hemos adoptado porque la creemos (hasta cierto punto) correcta. Por lo tanto, no tiene sentido ser tolerante con alguna doctrina moral que se niega a escuchar nuestra doctrina moral o interfiere en nuestras prácticas. 
Ahora bien, este límite posee una característica muy importante, que puede resultar también muy problemática: siempre se refieren a límites de otros individuos hacia uno. Un individuo racional tendrá entonces una razón externa para imponer límites a ciertas conductas de otros individuos, siempre que estas conductas afecten de algún modo las propias prácticas, conductas o el desarrollo de la propia doctrina. Pero no parece tener una razón externa para imponer límites cuando otros individuos realizan conductas hacia terceros, aun cuando, si esas conductas fueran dirigidas hacia uno, uno no las toleraría. Supongamos que un individuo $A$ adhiere y practica la doctrina $X$, que constituye su concepción comprehensiva del bien. $B$, en cambio, adhiere y practica la doctrina $Y$. Ahora bien, $A$ es racional, por lo cual acepta las dificultades del juzgar, por lo cual acepta el falibilismo ético, por lo cual es, prima facie, tolerante frente a $B$. $B$, por el contrario, posee una actitud dogmática respecto de $Y$ : cree $Y$ fervientemente y desea imponérsela a $C$. En este caso, parece que $A$ no tiene una razón externa para limitar su tolerancia frente a $B$, prohibiéndole (o intentando prohibirle) que sea intolerante frente a $C$. O para poner el problema de un modo más concreto: ¿Hasta qué punto estaría dispuesto un individuo externamente tolerante a tolerar sectas que resultan absolutamente intolerantes respecto de sus miembros, pero que a ese individuo no lo afectan ni directa ni indirectamente?

$\mathrm{Al}$ menos dos argumentos muestran que esto no es tan plausible. El primero es el siguiente. Ubiquémonos en el punto de vista de este individuo. Sabemos que deseamos practicar nuestra concepción comprehensiva del bien; también tenemos una posición no dogmática respecto de ella, lo cual, como hemos visto, nos lleva a ser tolerantes con puntos de vistas diferentes. Esto significa que sabemos que deberemos coexistir con individuos, eventualmente, grupos muy numerosos, que practican, adhieren, defienden, con- 
cepciones comprehensivas diferentes e incompatibles con la nuestra. Si bien estamos dispuestos a aceptar esta pluralidad, es cierto también que ella representa cierto peligro, en la medida en que algunos o muchos de estos grupos pueden no tener la actitud tolerante (dado que pueden no ser racionales). Esto quiere decir que estaremos interesados en fijar reglas que impidan actitudes intolerantes hacia nosotros. Ahora bien, sabemos (aquí está la clave de este argumento) que estas reglas (típicamente reglas jurídicas) sólo pueden ser generales. Si no lo fueran, no tendrían fuerza obligatoria. Sería sumamente inestable una legislación que permitiera, por ejemplo, la intolerancia dentro de una determinada secta o grupo social: una vez que la actitud intolerante se instala en determinados grupos, no existe seguridad de que uno mismo no será el próximo destinatario de esa actitud.

El segundo argumento apela al valor instrumental de la pluralidad. Supongamos que $A$ permite que $B$ sea intolerante frente a $C$, dado que esa actitud no lo afecta directamente. Además del peligro eventual que esto encierra, es evidente que el universo de doctrinas comprehensivas es más pequeño que si $B$ fuera tolerante hacia $C$. Y dado que $A$ está interesado en encontrar la doctrina correcta, le interesará poder escuchar a $C$ y observar sus prácticas.

Ahora bien, suponiendo que estos dos argumentos sean plausibles, ¿qué implican, desde el punto de vista sustantivo? Es decir, ¿qué contenido normativo poseería el consenso sobrepuesto de las diferentes doctrinas externamente tolerantes? Éste es un tema, sin duda, complejo y controvertido, pero en un sentido menos "peligroso" que el paso argumentativo anterior. Respecto de este problema puede argumentarse dentro de los límites de la racionalidad de medios y fines: cuál es el arreglo institucional o el conjunto de derechos que son el mejor instrumento para que puedan coexistir diferentes doctrinas comprehensivas 
del bien, puedan discutir públicamente, puedan ser oídas, ninguna pueda alcanzar la situación de hegemonía, etc. No es difícil suponer que el mejor instrumento consistirá, dicho muy someramente, en un conjunto de derechos básicos "intocables", tales como la libertad de expresión, de participación política, y, en general, los derechos garantizados en el primer principio de justicia de Rawls. Soy algo escéptico acerca de la posibilidad de ir más allá. La teoría de la justicia completa de Rawls (que incluye su segundo principio o principio de diferencia, su teoría de los bienes primarios, su teoría de la justicia intergeneracional, etc., etc.) es, en rigor, una doctrina muy articulada y plausible, pero comprehensiva, como lo es el utilitarismo, la moral católica, el marxismo (en alguna interpretación), u otras. Y como tal, no posee un "derecho especial" frente a las demás, sino sólo el derecho a ser escuchada con tolerancia.

\section{V}

Agrego ahora algunas reflexiones generales sobre la argumentación presentada.

Un punto central del argumento consiste, como se habrá notado, en inferir un principio de tolerancia o respeto o, en la terminología de Rawls, un pluralismo razonable, tomando como premisa el falibilismo ético, el cual, a su vez, se basa en las dificultades del juzgar. Si bien he distinguido este falibilismo ético del escepticismo ético, es necesario aceptar cierta similitud: en ambos casos, no podemos afirmar con total seguridad que nuestra doctrina moral es verdadera. El intento ya clásico de Kelsen de derivar la tolerancia del relativismo de los valores, ha sido, creo, criticado muy convincentemente (p.ej., por Carlos Nino): ${ }^{21}$ si todos los valores son relativos, entonces el valor de la tolerancia

${ }^{21}$ Nino 1989, pp. 52-54, donde cita a Kelsen 1975, pp. 40-41. 
también debería serlo. Aplicado a nuestro falibilismo ético, se podría sostener que, así como no estamos seguros de que nuestra doctrina comprehensiva de la moral sea verdadera, tampoco podríamos estar completamente seguros de que el pluralismo razonable y la tolerancia de diferentes doctrinas comprehensivas sean moralmente correctos. Y, en este sentido, continuaría la crítica, deberíamos aceptar la posibilidad de estar errados al respecto, lo cual implica que nuestro intento de fundamentación de una moral "política" es tan débil como los posibles intentos de fundamentación de las doctrinas morales comprehensivas. Creo, sin embargo, que con la argumentación presentada estamos en mejor posición para enfrentar este tipo de críticas de lo que lo estaba Kelsen. Es cierto que, tal vez, no podamos estar totalmente seguros de que la tolerancia de concepciones morales diferentes sea la posición "verdadera". Sin embargo, téngase presente que, si bien la tolerancia o el pluralismo razonable es una posición moral normativa, la razón que he delineado para aceptar esta posición no es una razón moral. El argumento es más bien condicional, y muy groseramente podría reconstruirse así: "Si usted está preocupado por saber qué es el bien, cuál es la vida buena, cuáles son los valores que debe perseguir, etc., y, además, existen razones para pensar que la tarea de averiguarlo es muy dificultosa, entonces usted debe ser tolerante y debe aceptar un pluralismo razonable de concepciones morales, religiosas, etc.". Este tipo de argumento no es accesible para el relativista "kelseniano" (es decir, tolerante). Por el contrario, el relativista kelseniano toma como premisa una posición que, en la terminología que introduje anteriormente, debería catalogarse como dogmática: él cree firmemente que no existen valores absolutos y que no tiene sentido preocuparse por buscar una fundamentación de tales valores. Por lo tanto, es perfectamente explicable, en el marco del argumento 
que yo he presentado aquí, que de esta posición no puedan extraerse consecuencias normativas tolerantes.

Mi idea respecto del escepticismo es, entonces, que debe tomarse como una teoría comprehensiva más, limitada por las dificultades del juzgar. En este sentido, si bien mi posición no se identifica con el escepticismo moral, es compatible con él (siempre que no sea dogmático). Podría pensarse, sin embargo, que mi posición es, en realidad, incompatible con el escepticismo, por otra razón. Si el razonamiento moral que he propuesto presupone que estamos preocupados por encontrar la doctrina moral verdadera, entonces toda la búsqueda sólo tiene sentido, si excluimos la posibilidad de que esta búsqueda no tenga ningún sentido. Para volver una vez más al paralelo con la ciencia, un presupuesto de la metodología científica que es hay verdad y falsedad, es decir, hay juicios verdaderos, aun cuando nunca se los pueda probar. En cambio, si nuestra doctrina es el escepticismo moral, entonces la búsqueda no tiene sentido, dado que presuponemos que no hay tal cosa como juicios morales verdaderos. Sin embargo, creo que esta crítica no se sostiene. La fundamentación de la tolerancia que he propuesto es compatible con el escepticismo, siempre que éste sea falibilista, es decir, que sostenga que no es posible la fundamentación de las normas, pero que no sea dogmático respecto de esta tesis. No tenemos por qué excluir de antemano la posibilidad de que toda la búsqueda por una teoría moral comprehensiva correcta sea inútil. Ésta es una posibilidad. Esta posibilidad no invalida el hecho de que tengamos un interés profundo por encontrar una teoría tal. Aceptar el escepticismo de un modo falibilista (es decir, aceptando que podemos estar equivocados al sostenerlo), nos obligará, si somos racionales, a escuchar los argumentos de aquellos que no son escépticos; y esto es el núcleo de la actitud tolerante que deseo defender. 
He dicho que el razonamiento condicional que resume mi argumentación es del tipo: si usted está preocupado por la verdad moral y no es dogmático (es decir, no cree haberla encontrado), entonces usted debe ser tolerante. Ahora bien, podría ser muy bien el caso de que alguien no esté preocupado en absoluto por este problema, que no le interese discutir, o podría ser que a alguien sí le interese el problema pero crea que ha encontrado la solución definitiva al respecto (es decir, sea dogmático). El argumento vale, obviamente, cuando ninguna de estas cosas se da, y, en este sentido, es un argumento relativo (relativo a ciertas premisas). Sin embargo, estas premisas son, creo, bastante plausibles, y no por razones estrictamente morales: ambas premisas pertenecen a lo que he denominado "racionalidad": es racional aceptar las dificultades del juzgar y es racional estar preocupados por encontrar la doctrina moral verdadera, dado que hacerlo daría sentido a nuestras vidas y a los fines que perseguimos. Lo primero nos lleva al falibilismo ético; lo segundo, a comprometernos a extraer consecuencias normativas de ello.

¿Qué ocurre, sin embargo, con aquellas personas que no satisfacen alguna de las dos premisas, es decir, que no están preocupadas por encontrar una doctrina moral, religiosa, o filosófica verdadera, o son dogmáticas (es decir, creen haberla encontrado)? Con tales personas no existe la posibilidad de diálogo o discusión, dado que, o bien no les interesa el tema, o bien ya creen haber encontrado la respuesta. Con ellas, la relación sólo puede ser de fuerza, no de argumentación: es racional tolerarlas, en la medida en que no se comporten de modo intolerante (es decir, en la medida en que sean internamente tolerantes), y utilizar la fuerza contra ellas, en el caso en que lo hagan. ${ }^{22}$

22 Existe cierta similitud estructural entre la argumentación que he presentado y la que N. Hoerster desarrolla en 1982. Allí él presenta 
La fundamentación de una doctrina política externamente tolerante es importante también para establecer las bases de la estabilidad (tal vez, la principal preocupación de Rawls en Political Liberalism). Creo que una sociedad tolerante sólo puede ser estable si son dominantes concepciones comprehensivas externamente tolerantes. La existencia de la tolerancia dentro de una sociedad dominada por doctrinas internamente tolerantes es una base insuficiente para la estabilidad, dado que ésta depende de una reflexión compleja y siempre discutible por parte de quienes defienden esas concepciones, acerca de cómo compatibilizar el dogmatismo con la tolerancia. Esto se refleja, por ejemplo, en las disputas entre adherentes al catolicismo acerca de si deben tolerar actitudes incompatibles con su religión (el ateísmo, la homosexualidad, el divorcio, las relaciones sexuales extramatrimoniales, los métodos anticonceptivos, etc.). Si bien existen católicos que son tolerantes frente a estas prácticas, existen otros que no lo son, y el equilibrio entre estas actitudes es muy inestable.

Una última reflexión acerca del alcance de la argumentación presentada. Aquí valen las restricciones que el propio Rawls impone a su argumentación: se trata de fundamentar una teoría "política", no comprehensiva. Esto quiere decir, una teoría a favor de ciertas instituciones básicas de la sociedad que regulen de un modo moralmente adecuado la convivencia entre individuos que adhieren a doctrinas morales, religiosas y filosóficas comprehensivas diferentes e incompatibles entre sí. No se trata, por tanto, de una argumentación que fundamente una moral en general, ni

un argumento que también se basa en ciertos intereses básicos para arribar a ciertas conclusiones morales. Por ejemplo, se basa en el interés extendido en no ser matado para abogar por la norma que prohibe matar. En mi caso, el interés es más abstracto y general (arribar a una doctrina moral correcta), lo cual, sostengo, me permite llegar a una norma de tolerancia también más general. 
que ofrezca razones para actuar moralmente. No responde a la pregunta del tonto debida a Hobbes: “ipor qué debo actuar moralmente, aun cuando no me conviene?". Sólo intenta probar que individuos racionales en el sentido amplio que define Rawls, es decir, individuos que adhieren a, o están preocupados por encontrar una determinada doctrina comprehensiva (entre las cuales puede incluirse el escepticismo moral), pero aceptan las dificultades del juzgar y sus consecuencias teóricas, tendrán razones para ser razonables, es decir, para aceptar la convivencia tolerante de diferentes estilos de vida no intolerantes y la implantación de los derechos e instituciones que ello implica. ${ }^{23}$

\section{BIBLIOGRAFÍA}

Barry, B., "John Rawls and the Search of Stability", Ethics, 105, 1995.

Dworkin, R., "Objectivity and Truth: You'd Better Believed It", Philosophy \& Public Affairs, 25, 2, 1996.

Hoerster, N., "Rechtsethik ohne Metaphysik", Juristenzeitung, 8, 1982.

Kelsen, H., Was ist Gerechtigkeit?, Franz Deutlicke, Viena, 2da. ed. 1975.

Mill, J.S., On Liberty, en Utilitarianism, On Liberty, Essay on Bentham, together with selected Writings of J. Bentham, Collins, Londres, 1970.

23 Versiones previas de este trabajo fueron leídas en el Departamento de Filosofía del Derecho de la Universidad Pompeu Fabra de Barcelona, en la Escuela de Derecho de la Universidad Torcuato Di Tella de Buenos Aires, en el Coloquio de Filosofía Práctica de la Sociedad Argentina de Análisis Filosófico y en el Departamento de Filosofía de la Universidad de Salamanca. En todos estos lugares he recibido comentarios que me han ayudado enormemente. Agradezco especialmente a Marcelo Alegre, Fernando Broncano, Ricardo Caracciolo, Martín Farrell, Victor Ferreres, Daniel Mendonca, Guido Pincione, Carlos Rosenrkantz, Horacio Spector, Neus Torbisco e Ignacio Zuberbühler. 
Nino, C., Ética y derechos humanos, Astrea, Buenos Aires, 2da. ed. 1989.

Popper, K., Logic of Scientific Discovery, Science Editions, Nueva York, 1961.

Rawls, J., Political Liberalism, Columbia University Press, Nueva York, 1993.

Wenar, L., "Political Liberalism: An Internal Critique", Ethics, 106, octubre 1995.

Recibido: 1 de septiembre de 1997 


\section{SUMMARY}

The main argument of this paper tries to justify some duties of respect and tolerance common in liberal societies. The argument proceeds in four steps. First, it shows that a rational person (in a broad sense) will take seriously into account the Rawlsian "burdens of judgment". The second step tries to prove that taking the burdens of judgment seriously leads a rational person to a fallibilist position regarding moral doctrines (in the sense of assuming that the own comprehensive moral view can be wrong). The third (and crucial) step of the argument aims to derive a tolerant attitude toward other (incompatible) conceptions of the good from the fallibilist position. And, finally, some limits of this attitude based on the same fallibilist position are considered and discussed. 